\title{
Critical appraisal of once-weekly formulation of exenatide in the control of type 2 diabetes mellitus
}

This article was published in the following Dove Press journal:

Diabetes, Metabolic Syndrome and Obesity:Targets and Therapy

15 April 2010

Number of times this article has been viewed

\author{
Jason Seewoodhary' \\ Leanne Griffin' \\ Stephen C Bain' \\ 'Department of Diabetes and \\ Endocrinology, Swansea School \\ of Medicine, University of Wales, \\ Swansea SA2 8PP, UK
}

Correspondence: Jason Seewoodhary Department of Diabetes and Endocrinology, Swansea School of Medicine, University of Wales, The Grove Building, Swansea SA2 8PP, UK Tel +44 01792205666

Email seewoodharyj@hotmail.com

\begin{abstract}
Exenatide (also known as exendin-4) is a glucagon-like peptide-1 mimetic, which is indicated for the treatment of type 2 diabetes mellitus. The currently available formulation of this drug is a twice-daily exenatide (exenatide BID) injection that should be administered within 60 minutes of food. Once-weekly exenatide (exenatide QW) formulation is now being assessed in a clinical trial program. Exenatide QW has been shown to be the only noninsulin monotherapy to achieve glycosylated hemoglobin levels of $<7 \%$ in $>75 \%$ of treated patients. It has also demonstrated potential cardiovascular benefits by lowering total and low-density lipoprotein cholesterol concentrations, triglyceride levels, and both systolic and diastolic blood pressure. In addition, patients treated with exenatide QW achieved significant weight loss, which may also lead to significant cardiovascular risk reduction. Exenatide QW is associated with a lower incidence of gastrointestinal adverse effects compared with exenatide BID, and no patients treated with exenatide QW monotherapy experienced a confirmed hypoglycemic event. Exenatide QW results in 24-hour coverage with exenatide concentrations that are known to improve glycemic control and remain well tolerated in patients with type 2 diabetes mellitus. This review defines the state of play with exenatide QW by critically appraising its role in clinical practice.
\end{abstract}

Keywords: GLP-1 mimetic, $\mathrm{HbA}_{1 \mathrm{c}}$, weight loss

\section{Introduction}

Type 2 diabetes mellitus is currently recognized as one of the main threats to human health worldwide, with the prevalence increasing in an epidemic fashion. ${ }^{1,2}$

General management issues in type 2 diabetes mellitus include education, diet, exercise, and weight loss. The latest treatment recommendations ${ }^{3}$ suggest a target glycosylated hemoglobin $\left(\mathrm{HbA}_{1 \mathrm{c}}\right)$ level $\leq 7 \%$, which has been shown to reduce microvascular complications of type 1 diabetes mellitus. Long-term follow-up of cohorts in the Diabetes Control and Complications Trial ${ }^{4}$ and UK Prospective Diabetes Study ${ }^{5}$ suggests that the treatment to $\mathrm{HbA}_{1 \mathrm{c}}$ targets at this level in the years soon after the diagnosis of diabetes mellitus is associated with long-term reduction in the risk of macrovascular disease. Until more evidence becomes available, the general target level of $<7 \%$ appears reasonable for most adults with type 2 diabetes mellitus.

Despite the availability of many antidiabetic agents, approximately $60 \%$ of individuals with diabetes mellitus do not achieve the target $\mathrm{HbA}_{1 \mathrm{c}}$ levels. ${ }^{6}$ The reasons for this include noncompliance, side effects of treatments, hypoglycemia, weight gain, problems with dose titration of antidiabetic agents, and changing and more stringent target $\mathrm{HbA}_{1 \mathrm{c}}$ levels by national healthcare organizations. Clearly, additional therapeutic 
options are needed, preferably the ones that will further improve glycemic control, reduce weight, and overcome other clinical shortcomings of available agents, especially hypoglycemia.

Incretin therapeutics are a relatively novel field and much remains to be discovered. This review examines the potential role of once-weekly exenatide (exenatide QW) formulation in the control of type 2 diabetes mellitus.

\section{The incretin system, glucagon-like peptide- I, and exenatide}

Incretins are a group of hormones released into the bloodstream from gastrointestinal $\mathrm{L}$ and $\mathrm{K}$ cells in response to a nutrient load. ${ }^{7}$ They cause a postprandial increase in the amount of insulin released from the $\beta$-cells of the islets of Langerhans even before blood glucose levels become elevated but act in a glucose-dependent manner, thereby limiting the risk of hypoglycemia. ${ }^{7}$ Incretins have other benefits on glucose metabolism. ${ }^{7}$ They slow the rate of absorption of nutrients into the bloodstream by reducing gastric emptying; reduce satiety, which aids weight loss; and inhibit glucagon release from the $\alpha$-cells of the islets of Langerhans. ${ }^{7}$ The two molecules that are recognized to have incretin effects are glucagonlike peptide-1 (GLP-1) and gastric inhibitory peptide (also known as glucose-dependent insulinotropic peptide [GIP]). ${ }^{7}$ Postprandial augmentation of insulin secretion by GLP-1 and GIP - the so-called incretin effect - is reduced in patients with type 2 diabetes mellitus. ${ }^{8}$ However, in patients with newly diagnosed type 2 diabetes mellitus with relatively good glycemic control $\left(\mathrm{HbA}_{1 \mathrm{c}}\right.$ levels of approximately $6.9 \%$ ), both GIP secretion and GLP-1 secretion in response to glucose and mixed-meal challenges are the same or increased when compared with healthy subjects. ${ }^{9}$ Furthermore, in patients with long-standing type 2 diabetes mellitus with relatively poor glycemic control $\left(\mathrm{HbA}_{1 \mathrm{c}}\right.$ levels of approximately $8 \%-9 \%$ ), the GLP-1 secretion is decreased, whereas the GIP secretion is unchanged. ${ }^{10}$ However, exogenous GLP-1 administration at pharmacological doses increases insulin secretion to normal levels and decreases plasma glucose effectively. ${ }^{11}$ In contrast, exogenous GIP administration, even at supraphysiological doses, has markedly reduced insulinotropic actions, with little or no glucose-lowering effects in patients with type 2 diabetes mellitus. ${ }^{11}$ Therefore, therapeutic strategies for type 2 diabetes mellitus are focused on the use of GLP-1 analogs, GLP-1 receptor (GLP-1R) agonists or GLP-1 mimetics, and not on the use of GIP.

The major drawback of using GLP-1 in the treatment of type 2 diabetes mellitus is its short half-life of approximately
2 minutes. ${ }^{12}$ GLP-1 is rapidly degraded by dipetidyl peptidase-4 (DPP-4), which cleaves the N-terminal dipeptides (His7-Ala8) from GLP-1 (7-36) and renders the resulting major metabolite GLP-1 (9-36) inactive. ${ }^{13}$ In addition, neutral endopeptidase 24.11 hydrolyzes GLP-1 at six different moieties. ${ }^{14}$ Because of such a short half-life, bolus subcutaneous injections of GLP-1 result in only a transient effect on insulin secretion and plasma glucose levels. ${ }^{15}$

The potential of using GLP-1-based therapy for type 2 diabetes mellitus has been realized by the development of GLP-1 mimetics; these mimetics are resistant to DPP-4 activity and thus have a longer half-life relative to endogenous GLP-1.

Exenatide, an incretin mimetic, is licensed as an adjunctive therapy in patients with type 2 diabetes mellitus who take metformin and/or a sulphonylurea but who do not achieve adequate glycemic control. ${ }^{15}$ It offers a wide range of beneficial glucoregulatory effects, including enhancement of glucose-dependent insulin secretion, restoration of first-phase insulin response, and suppression of inappropriately elevated glucagon secretion, thereby achieving $\mathrm{HbA}_{1 \mathrm{c}}$ level reductions of $0.6 \%-0.8 \% .{ }^{15}$ These effects were more pronounced earlier on in patients with diabetes mellitus and were attenuated if exenatide therapy was started at a later stage when the $\beta$-cell mass relatively depleted. ${ }^{15}$ By slowing gastric emptying and promoting satiety, exenatide also facilitates weight loss, which is important in obese patients in whom insulin may promote weight gain and worsen insulin resistance. ${ }^{16}$

Exenatide shares $53 \%$ amino acid homology to human GLP-1 and binds directly to GLP-1R. ${ }^{17}$ It binds more avidly to GLP-1R than to GLP-1 in GLP-1R-expressing cells. ${ }^{17}$ Placebo-controlled ${ }^{18}$ and comparator-controlled ${ }^{19}$ clinical studies have demonstrated that exenatide improves glycemic control and reduces body weight in patients with type 2 diabetes mellitus. These studies also showed improvement of surrogate measures of $\beta$-cell function. ${ }^{19}$ In addition, animal studies suggested that exogenous GLP-1 has the ability to increase islet size, enhance $\beta$-cell proliferation, inhibit $\beta$-cell apoptosis, and regulate islet growth. ${ }^{15}$ These effects are important because they increase the possibility that GLP-1 therapies may modify the progression of type 2 diabetes mellitus.

Short-acting exenatide (twice-daily exenatide [exenatide $\mathrm{BID}])$ has been shown to be effective in lowering $\mathrm{HbA}_{1 \mathrm{c}}$ level in a 30-week, randomized, AMIGO trials, which are summarized in Table 1.

Exenatide BID is administered by subcutaneous injection before each of the two main meals of the day, but may not provide complete coverage overnight and of midday meals. ${ }^{16}$ 
Table I Summary of the AMIGO trials

\begin{tabular}{|c|c|c|c|c|c|}
\hline Trial & $\begin{array}{l}\text { No. of } \\
\text { participants }\end{array}$ & $\begin{array}{l}\text { Exenatide }+[\text { drug }] \\
\text { versus placebo }+[\text { drug }]\end{array}$ & $\begin{array}{l}\text { Effect of } 5 \mu \mathrm{g} \text { exenatide } \\
\text { on } \mathrm{HbA} \mathrm{A}_{\mathrm{lc}}\end{array}$ & $\begin{array}{l}\text { Effect of } 10 \mu \mathrm{g} \\
\text { exenatide on } \mathrm{HbA}_{\mathrm{lc}}\end{array}$ & $\begin{array}{l}\text { Effect of placebo } \\
\text { on } \mathrm{HbA} \mathrm{A}_{\mathrm{Ic}}\end{array}$ \\
\hline AMIGO-I 20 & 336 & Metformin & $0.4 \%$ reduction & $0.8 \%$ reduction & $\begin{array}{l}0.1 \% \text { increase }(P<0.002 \\
\text { vs placebo for both } \\
\text { exenatide groups) }\end{array}$ \\
\hline AMIGO-2 ${ }^{19}$ & 377 & Sulphonylurea & $0.5 \%$ reduction & $0.9 \%$ reduction & $\begin{array}{l}0.1 \% \text { increase }(P<0.001 \\
\text { vs placebo for both } \\
\text { exenatide groups) }\end{array}$ \\
\hline AMIGO- $3^{21}$ & 733 & $\begin{array}{l}\text { Metformin and } \\
\text { sulphonylurea }\end{array}$ & $0.6 \%$ reduction & $0.8 \%$ reduction & $\begin{array}{l}0.2 \% \text { increase }(P<0.001 \\
\text { vs placebo for both } \\
\text { exenatide groups) }\end{array}$ \\
\hline
\end{tabular}

Therefore, exenatide QW formulation has been developed. Exenatide QW incorporates a delivery technology that uses biodegradable polymeric microspheres to entrap exenatide and provide extended release of the medication. ${ }^{22}$ Once injected into the subcutaneous tissues, the microspheres biodegrade over time, thereby releasing the bioactive molecules in a controlled manner. ${ }^{22}$

The time to peak plasma and steady-state concentrations is increased with exenatide QW relative to exenatide BID. ${ }^{22}$ Exenatide concentrations that significantly reduce fasting plasma glucose are achieved within 2 weeks of exenatide QW dosing, and plateau concentrations are achieved within 6-10 weeks. ${ }^{22}$ The range of steady-state concentrations for exenatide QW encompasses the peak plasma concentrations observed for exenatide BID. ${ }^{22}$ As a result, exenatide QW results in 24-hour coverage with exenatide concentrations that are known to improve glycemic control and remain well tolerated in patients with type 2 diabetes mellitus. ${ }^{22}$

\section{Efficacy of exenatide QW}

Controlled clinical trials have demonstrated multiple effects of exenatide QW on glycemic control in patients with type 2 diabetes mellitus. A small, dose-ranging clinical trial ${ }^{22}$ in patients unable to maintain adequate glycemic control with either diet and exercise alone or metformin monotherapy showed significant $\mathrm{HbA}_{1 \mathrm{c}}$ level reductions and weight loss with exenatide QW. The trial consisted of a 15-week treatment phase, followed by a 12 -week safety follow-up period, during which no drug was administered. Patients were randomized to placebo, $0.8 \mathrm{mg}$ exenatide $\mathrm{QW}$, and $2 \mathrm{mg}$ exenatide $\mathrm{QW}$. The study population had a mean $\mathrm{HbA}_{1 \mathrm{c}}$ level of $8.5 \%$ and a mean disease duration of 5 years. At both does, exenatide QW resulted in an improved fasting plasma glucose and postprandial glycemic control and a significant reduction in $\mathrm{HbA}_{1 \mathrm{c}}$ level, with final $\mathrm{HbA}_{1 \mathrm{c}}$ levels of $7.2 \%$ and $6.6 \%$ for 0.8 and $2 \mathrm{mg}$ exenatide QW, respectively. Approximately $80 \%$ of the patients treated with $2 \mathrm{mg}$ exenatide QW achieved an $\mathrm{HbA}_{1 \mathrm{c}}$ level of $<7 \%$, and there were significant reductions in body weight, with an average weight loss of $3.8 \mathrm{~kg}$ (compared with no weight loss in those who received $0.8 \mathrm{mg}$ exenatide QW and placebo). However, this study was limited by the small sample size $(n=45)$.

In support of these findings, $2 \mathrm{mg}$ exenatide QW demonstrated significant reductions in $\mathrm{HbA}_{1 \mathrm{c}}$ levels compared with $10 \mu \mathrm{g}$ exenatide BID at 30 weeks in the DURATION-1 (Diabetes therapy Utilisation: Research changes in $\mathrm{HbA}_{1 \mathrm{c}}$, weight and other factors $T$ hrough Intervention with exenatide Once weekly) study that included 295 patients with type 2 diabetes mellitus. ${ }^{23}$ At 30 weeks, the patients given exenatide QW had significantly greater reductions in $\mathrm{HbA}_{1 \mathrm{c}}$ levels than those given exenatide BID $(P=0.0023)$. A significantly greater proportion of patients receiving exenatide $\mathrm{QW}$ compared with patients receiving exenatide BID achieved target $\mathrm{HbA}_{1 \mathrm{c}}$ levels of $<7 \%$ ( $77 \%$ vs $\left.61 \%, P=0.0039\right)$, with no increased risk of hypoglycemia.

DURATION-1 study ${ }^{23}$ also reported important changes in cardiovascular disease (CVD) risk factors. Compared with the exenatide BID regimen, patients receiving exenatide QW had greater reductions from baseline in mean total and low-density lipoprotein cholesterol concentrations $(-0.13 \%$ vs $+0.03 \%)$. However, the clinical significance of these reductions is not clear because the confidence intervals are very broad. Both groups experienced similar reductions in triglycerides from baseline, similar reductions in blood pressure, and similar weight loss. However, the results of DURATION-1 study ${ }^{23}$ are limited by the lack of long-term data, especially on exenatide QW's capacity to reduce diabetic complications.

Data from DURATION-2 study ${ }^{24}$ have shown $2 \mathrm{mg}$ exenatide QW to provide superior glycemic control and weight loss compared with sitagliptin (100 mg once daily [OD]) and pioglitazone (45 mg OD) in 491 patients taking stable doses of 
metformin. Patients randomized to exenatide QW achieved a significantly lower $\mathrm{HbA}_{1 \mathrm{c}}$ level from baseline (1.7\%) compared with those randomized to sitagliptin (1\%) and pioglitazone $(1.4 \%)$ at 26 weeks. The exenatide QW cohort also achieved statistically significant weight loss $(-2.8 \mathrm{~kg})$ compared with the sitagliptin $(-0.9 \mathrm{~kg})$ and pioglitazone $(+3.4 \mathrm{~kg})$ cohorts at 6 months. In addition, DURATION- 2 study $^{24}$ demonstrated that exenatide $\mathrm{QW}$ achieved target $\mathrm{HbA}_{1 \mathrm{c}}$ levels, with no episodes of major hypoglycemia.

The unpublished DURATION-3 trial has compared the efficacy of $2 \mathrm{mg}$ exenatide QW with insulin glargine administered OD in 467 patients with type 2 diabetes mellitus treated with metformin alone or in combination with a sulphonylurea. Both treatment arms started with a baseline $\mathrm{HbA}_{1 \mathrm{c}}$ level of $8.3 \%$, and exenatide QW provided statistically significantly greater $\mathrm{HbA}_{1 \mathrm{c}}$ reductions, weight loss versus weight gain, and fewer episodes of hypoglycemia compared with insulin glargine administered OD. Subjects in both treatment groups are continuing in an open-ended extension study. Results are expected in 2010 .

In an ongoing randomized, double-blind trial, the efficacy of $2 \mathrm{mg}$ exenatide QW is being compared against that of metformin, sitagliptin, and pioglitazone as monotherapy in 822 drug-naive patients with type 2 diabetes mellitus (DURATION-4). The results are expected this year.

DURATION-5 study is a 26-week, 240-subject substudy designed to establish exenatide QW's superiority over exenatide BID. The results are expected in 2010.

In summary, these efficacy data demonstrate that exenatide QW results in significantly improved glycemic control and targets multiple aspects of type 2 diabetes mellitus. Exenatide QW has the additional therapeutic advantages of greater fasting glucose and glucagon reductions and greater $\mathrm{HbA}_{1 \mathrm{c}}$ level reductions by achieving constant and higher plasma drug concentrations.

\section{Safety of exenatide QW}

The most common side effects associated with exenatide BID include nausea, vomiting, and diarrhea, which are dose dependent and more common during drug initiation but subside over time. ${ }^{15}$

The risk of hypoglycemia is increased in patients on exenatide BID who also take a sulphonylurea, ${ }^{19}$ whereas it is not increased when exenatide BID is combined with metformin or a thiazolidiedione. ${ }^{25}$

Cases of acute pancreatitis have been reported with exenatide $\mathrm{BID},{ }^{26}$ but these are rare, and recent estimates indicate an incidence of $0.33-0.44$ per 1,000 adults per year. ${ }^{27}$ Furthermore, the pancreatitis risk may be increased in patients with type 2 diabetes mellitus compared with nondiabetic patients, suggesting that the risk is related to the underlying disease state rather than being a complication of exenatide therapy. ${ }^{27}$ If pancreatitis is suspected, then exenatide treatment should be discontinued. ${ }^{26}$

Exenatide, being a synthetic version of a nonmammalian hormone, has been associated with the development of antiexenatide antibodies when given as exenatide BID. ${ }^{20}$ However, the presence of antiexenatide antibodies had no predictive effect on glycemic control or adverse events. ${ }^{20}$

Recently, the Food and Drug Administration (FDA) issued a safety warning on exenatide BID, associating it with the development of renal failure. ${ }^{28}$ This FDA warning was based on 78 postmarketing cases reported between April 2005 and October 2008. The average patient age in the case reports was 60 years. The FDA approved revisions to the drug label for exenatide because of the temporal - causal relationship that was observed with the initiation of exenatide and the occurrence of serious potential consequences of altered kidney function. Of the 78 cases reported, 62 cases were classified as acute renal failure and the remaining 16 cases were classified as renal insufficiency. The postmarketing surveillance data of these patients reported that after the initiation of exenatide, hospitalization was required in $91 \%(71 / 78)$ of the patients. Kidney transplantation was required in 2 patients, and there were 4 fatalities. Dialysis had to be initiated in 18 patients, of which 2 patients had a known history of altered kidney function. Upon discontinuation, 39 patients reported improved signs and symptoms, and recurrence of kidney dysfunction was reported in 1 patient who was reinitiated on exenatide BID.

In contrast to exenatide BID, exenatide QW appears to have a lower incidence of side effects. In DURATION-1 study, ${ }^{23}$ exenatide QW was associated with a lower incidence of nausea compared with exenatide BID (26\% vs 35\%, respectively). Furthermore, patients on exenatide QW had a lower incidence of vomiting (11\% vs 19\%), and only 2 out of 148 patients on exenatide QW discontinued treatment because of these side effects. ${ }^{23}$

In DURATION-1 study, ${ }^{23}$ no patients on exenatide QW had a hypoglycemic episode if their background therapy did not include a sulphonylurea. With concurrent sulphonylurea use, $15 \%$ of patients reported a minor hypoglycemic event, similar to patients on exenatide BID. No major hypoglycemic episodes were reported.

There are currently no data on the incidence or risk of pancreatitis associated with exenatide QW. However, concerns about pancreatitis only surfaced long after FDA 
approval of exenatide BID. Of specific concern is the additional risk related to exenatide $\mathrm{QW}$ 's persistence after injection. For example, if pancreatitis occurs in an exenatide QW recipient, would it worsen over time because the drug takes 3 weeks to wash out of the body, compared with 1 day for exenatide BID?

Injection site reactions have been associated with exenatide QW, consisting of small subcutaneous nodules, which may be accompanied by pain, induration, erythema, or bleeding. ${ }^{29}$ Injection site pruritus is thought to be due to in vivo degradation of sustained-release delivery systems. ${ }^{30}$ This was observed in $18 \%$ of patients on exenatide QW in DURATION-1 study ${ }^{23}$ and was of mild intensity, which improved and then resolved with further exenatide treatment. ${ }^{23}$

Similar to patients on exenatide BID, antiexenatide antibodies have been observed in patients on exenatide $\mathrm{QW}$, but antibodies were of low titers $(<1 / 625) .{ }^{23}$ DURATION-1 study found that antiexenatide antibody titers were higher with the once-weekly dosing rather than the twice-daily dosing $(P=0.0002)$. The significance of this finding is not clear. Antiexenatide antibodies have no predictive effect on glycemic control with exenatide BID, whereas there is no evidence to date as to how they may affect glycemic control with exenatide $\mathrm{QW}{ }^{23}$

The FDA has expressed specific concerns about cardiovascular risk associated with new diabetes medicines. In this regard, exenatide $\mathrm{QW}$ is relying heavily on a meta-analysis of its parent compound, exenatide BID, that showed a reduced CVD risk of 0.70 compared with controls. ${ }^{31}$ However, the main limitation of this study is the open-label, uncontrolled nature of the study design, which does not provide a placebo group for comparison.

\section{Role of exenatide QW in clinical practice}

There is emerging evidence that exenatide QW will have major effects on patient-focused perspectives such as quality of life, satisfaction, and compliance. ${ }^{32}$ In addition, patient-reported measures can be used to examine whether drug differences other than clinical efficacy have an impact on outcomes that may be important to patients.

Exenatide QW may result in improved patient compliance compared with exenatide BID. Not surprisingly, patients seem to prefer taking a medication once a week rather than twice a day, suggesting exenatide QW would show improved compliance compared with exenatide BID. This simpler regimen could improve treatment adherence in real-world clinical settings. ${ }^{33}$
In a randomized, open-labeled, multicenter study, ${ }^{32}$ treatment satisfaction and weight-related quality of life in patients with type 2 diabetes mellitus treated with either exenatide BID or exenatide QW were assessed over 1 year. Exenatide QW was shown to significantly reduce the perceived frequency of hyperglycemia, ie, osmotic symptoms, and improve overall patient satisfaction with continued treatment compared with exenatide BID. A quality-of-life analysis revealed that patients reported higher overall treatment satisfaction with either exenatide BID $(n=147)$ or exenatide QW $(n=148)$ compared with their baseline therapy. Furthermore, in this study, treatment satisfaction and quality of life improved significantly for those switching from exenatide BID to exenatide QW. This study had the advantage of having a large sample size $(n=295)$ and a relatively long duration (52 weeks) to be able to detect clinically significant differences. In addition, the study outcomes, treatment satisfaction and quality of life, were assessed using validated questionnaires likely to be sensitive to the established clinical effects of the study medication. However, findings from this study were limited by the fact that there was no placebo group that continued with its prestudy medications to assess placebo effects and there was no crossover from exenatide QW to exenatide BID to assess order effects. Also, the participation of ethnic minorities was low, limiting the generalizability of the study findings to these groups, and the study did not examine outcomes for psychological well-being and diabetes distress for patients on exenatide QW.

Patient satisfaction for exenatide BID has been compared with that for insulin glargine in a randomized, 26-week international trial that studied 549 patients with type 2 diabetes mellitus. ${ }^{34}$ This trial found that both exenatide and insulin glargine were associated with significant improvements in patient-reported outcomes when added to oral medications among patients with type 2 diabetes mellitus. Despite an additional daily injection and a higher rate of gastrointestinal adverse events, treatment satisfaction of the exenatide group was comparable to that of the insulin glargine group, possibly because of weight loss observed in patients treated with exenatide. This trial ${ }^{34}$ had the advantage of using validated multiple scoring systems (the Vitality Scale of the SF-36, the Diabetes Symptom Checklist - Revised, the EuroQol EQ-5D, the Treatment Flexibility Scale, and the Diabetes Treatment Satisfaction Questionnaire) to accurately assess patient outcome measures, which would increase the reliability of the findings. However, findings from the study were limited by a lack of generalizability as the study population was predominantly Caucasian, with slightly more men than women. 
In a randomized, controlled trial, ${ }^{35}$ the long-term clinical and economic outcomes associated with exenatide BID were compared with those associated with insulin glargine as "add-on" treatments to oral therapy in patients with type 2 diabetes mellitus inadequately controlled with combination oral hypoglycemic agents in a computer simulation model of diabetes mellitus. This was used to project complications, life expectancy, quality-adjusted life expectancy, and direct medical costs over a 35-year time horizon. Exenatide BID was associated with comparable life expectancy and an improvement in quality-adjusted life expectancy versus insulin glargine over a 35-year time horizon. Based on current standards, exenatide BID would be a cost-effective, alternative treatment to insulin glargine for patients with type 2 diabetes mellitus inadequately controlled with oral therapy. However, the findings of this study are limited by a lack of both reliability and validity to a real-world clinical environment.

Unlike exenatide BID, patient satisfaction with exenatide QW has not yet been compared with that with insulin glargine in a head-to-head study. However, as exenatide QW has a better gastrointestinal adverse effect profile than exenatide BID, one would expect improved patient satisfaction with exenatide QW compared with insulin glargine. Unlike exenatide BID, there are no economic or health outcome data comparing exenatide QW with insulin glargine.

There is some evidence from a murine model of nonalcoholic steatohepatitis (NASH) that exenatide BID can reverse hepatic steatosis. ${ }^{36}$ In a preliminary study ${ }^{37}$ on 31 patients for over 2 years, exenatide BID treatment led to a decrease in hepatic transaminase enzymes independent of weight loss. In a multicenter, double-blind, placebo-controlled trial $^{38}$ of 974 patients with type 2 diabetes mellitus, it was found that adjunctive exenatide treatment for 2 years resulted in a reduction in hepatic injury biomarkers, AST and ALT. Based on this observation, an as yet unpublished clinical trial sponsored by the National Institute of Diabetes and Digestive and Kidney Diseases is under way, looking at whether exenatide BID administered subcutaneously for 24-28 weeks improves liver histology in diabetic patients with biopsyproven NASH. In contrast, there are no data on the potential disease-modifying role of exenatide QW in reversing NASH, and further research is needed in this area.

\section{Other long-acting GLP-I mimetics}

Other GLP-1 mimetics that require less-frequent dosing than exenatide BID are currently available.

Liraglutide, a once-daily GLP-1 analog that has recently received FDA approval, was shown to enable many patients to achieve target $\mathrm{HbA}_{1 \mathrm{c}}$ levels and to improve several morbidities commonly associated with type 2 diabetes mellitus. It induced weight loss, reduced systolic blood pressure, and improved $\beta$-cell function. ${ }^{39}$ It was well tolerated, although an increased incidence of mild nausea was observed. ${ }^{39}$ It has recently been licensed in the USA, Europe, and Japan.

However, recent concerns about C-cell medullary thyroid cancer associated with liraglutide, which has led to a "black-box" warning, may also need to be characterized for exenatide QW before it is approved by the FDA. Liraglutide has been associated with thyroid hyperplasia and medullary thyroid carcinoma. This occurs through GLP-1 receptor stimulation of thyroid C-cells. ${ }^{39}$ As GLP-1 mimetics stimulate the same receptor in pancreatic $\beta$-cells that is also found in the C-cells of thyroid tissue, where the insidious medullary thyroid cancer forms, there is a theoretical risk that exenatide, being a related GLP-1 mimetic, may have a similar effect. However, rodent C-cell tumors induced by liraglutide are caused by a nongenotoxic, specific receptormediated mechanism, to which rodents are particularly sensitive and humans are not. ${ }^{40}$ To date, there is no evidence of a causal relationship between exenatide and human $\mathrm{C}$-cell medullary thyroid cancer.

Other GLP-1 mimetics include AVE0010, ${ }^{41}$ albiglutide, ${ }^{42}$ and taspoglutide. ${ }^{43}$ All are currently not licensed.

In a Phase IIb trial ${ }^{41}$ of 542 patients, AVE0010 was found to be well tolerated and significantly improve glycemic control versus placebo in patients with type 2 diabetes mellitus inadequately controlled with metformin alone. The once-daily regimen demonstrated a clear dose response with a $\mathrm{HbA}_{1 \mathrm{c}}$ level reduction similar to the reduction associated with the twicedaily regimen. AVE0010 was also found to be associated with weight loss and postprandial glucose reductions. ${ }^{41}$

Albiglutide is a novel DPP-4-resistant GLP-1 dimer fused to human albumin. ${ }^{42}$ In clinical trials, ${ }^{42}$ albiglutide improved fasting plasma glucose and postprandial glucose, with a favorable safety profile in subjects with type 2 diabetes mellitus. Albiglutide's long half-life may allow for once-weekly or less-frequent dosing.

In a double-blind, placebo-controlled study, ${ }^{43}$ taspoglutide, a once-weekly human GLP-1 analog, when combined with metformin significantly improves fasting and postprandial glycemic control and induces weight loss, with a favorable tolerability profile.

However, unlike exenatide QW, none of these compounds have consistently demonstrated stable and sustained drug concentrations over several days. 


\section{Conclusion}

All available treatments for type 2 diabetes mellitus have potential limitations such as daily administration and the need for dose titration. Adverse effects such as weight gain, fluid retention, gastrointestinal intolerability, and hypoglycemia have led to difficulty in achieving optimal control of type 2 diabetes mellitus. Given the rapidly increasing prevalence of diabetes mellitus and obesity worldwide, there is an urgent need for new, safe, and effective treatments, and incretin mimetics have emerged promising candidates that fulfil these criteria.

Exenatide BID is the first GLP-1 receptor agonist approved for the treatment of type 2 diabetes mellitus, and it lowers blood glucose by multiple mechanisms.

In clinical trials, exenatide $\mathrm{QW}$ has been shown to achieve better glycemic control compared with exenatide BID, with a lower incidence of adverse effects. Clinical trials have also demonstrated possible beneficial cardiovascular effects of exenatide QW. However, much remains to be discovered about this novel compound. Whether exenatide $\mathrm{QW}$ preserves $\beta$-cell mass and function and whether it has a disease-modifying effect in NASH warrant further research. By slowing gastric emptying and reducing satiety, exenatide $\mathrm{QW}$ has been shown to facilitate weight loss. Whether exenatide QW could be investigated as an antiobesity treatment in patients with no type 2 diabetes mellitus warrants further research.

\section{Disclosures}

The authors report no conflicts of interest in this work.

\section{References}

1. Zimmer P, Alberti KG, Shaw J. Global and societal implications of the diabetes epidemic. Nature. 2001;414:782-787.

2. Zimmet P, Lefèbvre P. The global NIDDM epidemic: treating the disease and ignoring the symptom [Editorial]. Diabetologia. 1996;39:1247-1248.

3. American Diabetes Association. Standards of medical care in diabetes 2009. Diabetes Care. 2009;32 Suppl 1:S13-S61.

4. The DCCT Research Group. The effect of intensive treatment of diabetes on the development and progression of long-term complications in insulin-dependent diabetes mellitus. N Engl J Med. 1993;329: 977-986.

5. Stratton IM, Adler AI, Neil HAW, et al. Association of glycaemia with macrovascular and microvascular complications of type 2 diabetes (UKPDS 35): prospective observational study. BMJ. 2000;321:405-412.

6. Saydah SH, Fradkin J, Cowie CC. Poor control of risk factors for vascular disease among adults with previously diagnosed diabetes. JAMA. 2004;291:335-342.

7. Kjems LL, Holst JJ, Vølund A, Madsbad S. The influence of GLP-1 on glucose-stimulated insulin secretion: effects on beta-cell sensitivity in type 2 and nondiabetic subjects. Diabetes. 2003;52:380-386.

8. Meier JJ, Nauck MA. Is secretion of glucagon-like peptide-1 reduced in type 2 diabetes mellitus? Nat Clin Pract Endocrinol Metab. 2008;4:606-607.

9. Vollmer K, Holst JJ, Baller B, et al. Predictors of incretin concentrations in subjects with normal, impaired, and diabetic glucose tolerance. Diabetes. 2008;57:678-687.
10. Vilsbøll T, Krarup T, Deacon CF, Madsbad S, Holst JJ. Reduced post-prandial concentrations of intact biologically active glucagon-like peptide 1 in type 2 diabetic patients. Diabetes. 2001;50:609-613.

11. Nauck MA, Heimesaat MM, Orskov C, Holst JJ, Ebert R, Creutzfeldt W. Preserved incretin activity of glucagon-like peptide 1 [7-36 amide] but not of synthetic human gastric inhibitory polypeptide in patients with type-2 diabetes mellitus. J Clin Invest. 1993;91:301-307.

12. Meier JJ, Nauck MA, Kranz D, et al. Secretion, degradation and elimination of glucagon-like peptide 1 and gastric inhibitory polypeptide in patients with chronic renal insufficiency and healthy control subjects. Diabetes. 2004;53:654-662.

13. Hansen L, Deacon CF, Orskov C, Holst JJ. Glucagon-like peptide-1(7-36)amide is transformed to glucagon-like peptide-1-(9-36)amide by dipeptidyly peptidase IV in the capillaries supplying the L cells of the porcine intestine. Endocrinology. 1999;140:5356-5363.

14. Hupe-Sodmann K, McGregor GP, Bridenbaugh R, et al. Characterisation of the processing by human neutral endopeptidase 24.11 of GLP-1(7-36) amide and comparison of the substrate specificity of the enzyme for other glucagon-like peptides. Regul Pept. 1995;58:149-156.

15. Verspohl EJ. Novel therapeutics for type 2 diabetes: incretin hormone mimetics (glucagon-like peptide-1 receptor agonists) and dipeptidyl peptidase-4 inhibitors. Pharmacol Ther. 2009;124:113-138.

16. Copley K, McCowen K, Hiles R, Nielsen LL, Young A, Parkes DG. Investigation of exenatide elimination and its in vivo and in vitro degradation. Curr Drug Metab. 2006;7:367-374.

17. Thorens B, Porret A, Bühler L, Deng SP, Morel P, Widmann C. Cloning and functional expression of the human islet GLP-1 receptor. Demonstration that exendin-4 is an agonist and exendin-(9-39) an antagonist of the receptor. Diabetes. 1993;42:1678-1682.

18. Brubaker PL, Drucker DJ. Minireview: glucagon-like peptides regulate cell proliferation and apoptosis in the pancreas, gut, and central nervous system. Endocrinology. 2004;145:2653-2659.

19. Buse JB, Henry RR, Han J, Kim DD, Fineman MS, Baron AD; for Exenatide-113 Clinical Study Group. Effects of exenatide (exendin-4) on glycemic control over 30 weeks in sulfonylurea-treated patients with type 2 diabetes. Diabetes Care. 2004;27:2628-2635.

20. DeFronzo RA, Ratner RE, Han J, Kim DD, Fineman MS, Baron AD. Effects of exenatide (exendin-4) on glycemic control and weight over 30 weeks in metformin-treated patients with type 2 diabetes. Diabetes Care. 2005;28:1092-1100.

21. Kendall DM, Riddle MC, Rosenstock J, et al. Effects of exenatide (exendin-4) on glycemic control over 30 weeks in patients with type 2 diabetes treated with metformin and a sulfonylurea. Diabetes Care. 2005;28:1083-1091

22. Kim D, MacConell L, Zhuang D, et al. Effects of once-weekly dosing of a long-acting release formulation of exenatide on glucose control and body weight in subjects with type 2 diabetes. Diabetes Care. 2007;30:1487-1493.

23. Drucker DJ, Buse JB, Taylor K, et al; for DURATION-1 Study Group. Exenatide once weekly versus twice daily for the treatment of type 2 diabetes: a randomised, open-label, non-inferiority study. Lancet. 2008;372:1240-1250.

24. Bergenstal R, Wysham C, Yan P, Macconell L, Malloy J, Porter L. DURATION-2: exenatide once weekly demonstrated superior glycemic control and weight reduction compared to sitagliptin or pioglitazone after 26 weeks of treatment. American Diabetes Association 2009 Scientific Sessions; 2009 Jun 5-9; New Orleans, LA, USA. Abstract 6-LB.

25. Zinman B, Hoogwerf BJ, Durán García S, et al. The effect of adding exenatide to a thiazolidinedione in suboptimally controlled type 2 diabetes: a randomised trial. Ann Intern Med. 2007;146:477-485.

26. Bain SC, Stephens JW. Exenatide and pancreatitis: an update. Expert Opin Drug Saf. 2008;7:643-644.

27. Noel RA, Braun DK, Patterson R, Bloomgren G. Estimated incidence of acute pancreatitis in patients with type 2 diabetes. Pharmacol Drug Saf. 2008;17:S1-S29.

28. Weise WJ, Sivanandy MS, Block CA, Comi RJ. Exenatide-associated ischemic renal failure. Diabetes Care. 2009;32:e22-e23. 
29. BYETTA (exenatide) injection prescribing information 2008.

30. Garbutt JC, Kranzler HR, O’Malley SS, et al; for Vivitrex Study Group. Efficacy and tolerability of long-acting injectable naltrexone for alcohol dependence: a randomised controlled trial. JAMA. 2005;293:1617-1625.

31. Klonoff DC, Buse JB, Nielsen LL, et al. Exenatide effects on diabetes, obesity, cardiovascular risk factors and hepatic biomarkers in patients with type 2 diabetes treated for at least 3 years. Curr Med Res Opin. 2008;24:275-286.

32. Best JH, Boye KS, Rubin RR, Cao D, Kim TH, Peyrot M. Improved treatment satisfaction and weight-related quality of life with exenatide once weekly or twice daily. Diabet Med. 2009;26(7):722-728.

33. Rubin RR. Adherence to pharmacologic therapy in patients with type 2 diabetes mellitus. Am J Med. 2005;118 Suppl 5A:S27-S34.

34. Secnik Boye K, Matza LS, Oglesby A, et al. Patient-reported outcomes in a trial of exenatide and insulin glargine for the treatment of type 2 diabetes. Health Qual Life Outcomes. 2006;4:80.

35. Brändle M, Erny-Albrecht KM, Goodall G, Spinas GA, Streit P, Valentine WJ. Exenatide versus insulin glargine: a cost-effectiveness evaluation in patients with type 2 diabetes in Switzerland. Int J Clin Pharmacol Ther. 2009;47:501-515.

36. Stein LL, Dong MH, Loomba R. Insulin sensitisers in nonalcoholic fatty liver disease and steatohepatitis: current status. Adv Ther. 2009;26:893-907.
37. Bulchandani D, Nachnani J, Eaton C, Hamburg M. Association of exenatide with liver enzymes in patients with type 2 diabetes. Endocrinologist. 2009;19:114-115.

38. Buse JB, Klonoff DC, Nielsen LL, et al. Metabolic effects of two years of exenatide treatment on diabetes, obesity, and hepatic biomarkers in patients with type 2 diabetes: an interim analysis of data from the openlabel, uncontrolled extension of three double-blind, placebo-controlled trials. Clin Ther. 2007;29:139-153.

39. Aimaretti G. Liraglutide: a once-daily human glucagon-like peptide-1 analogue. J Endocrinol Invest. 2009;32:701-703.

40. Croom KF, McCormack PL. Liraglutide: a review of its use in type 2 diabetes mellitus. Drugs. 2009;69:1985-2004.

41. Werner U. Preclinical pharmacology of the new GLP-1 receptor agonist AVE0010. Ann Endocrinol (Paris). 2008;69:164-165.

42. Rosenstock J, Reusch J, Bush M, Yang F, Stewart M; for Albiglutide Study Group. Potential of albiglutide, a long-acting GLP-1 receptor agonist, in type 2 diabetes: a randomized controlled trial exploring weekly, biweekly, and monthly dosing. Diabetes Care. 2009;32:1880-1886.

43. Nauck MA, Ratner RE, Kapitza C, Berria R, Boldrin M, Balena R. Treatment with the human once-weekly glucagon-like peptide-1 analog taspoglutide in combination with metformin improves glycemic control and lowers body weight in patients with type 2 diabetes inadequately controlled with metformin alone: a double-blind placebo-controlled study. Diabetes Care. 2009;32:1237-1243.

\section{Publish your work in this journal}

Diabetes, Metabolic Syndrome and Obesity: Targets and Therapy is an international, peer-reviewed open-access journal committed to the rapid publication of the latest laboratory and clinical findings in the fields of diabetes, metabolic syndrome and obesity research. Original research, review, case reports, hypothesis formation, expert opinion and commentaries are all considered for publication. The manuscript management system is completely online and includes a very quick and fair peer-review system, which is all easy to use. Visit http://www.dovepress.com/testimonials.php to read real quotes from published authors. 